\title{
Improved estimates of conduction velocity distributions using single unit action potentials
}

\author{
T E M IL NER, R B STEIN, J G I LLESPIE, A N D H A N L Y \\ From the Department of Physiology, University of Alberta, Edmonton, Canada
}

SUMMARY Single unit potentials were recorded from sural and medial gastrocnemius nerves. Action potential amplitude, integrated area and half-width (duration) were approximately proportional to conduction velocity, raised to the powers $1.5,1$ and -0.5 respectively for the sural nerve, and 2, 1.5 and -0.5 for the medial gastrocnemius nerve. These empirical relationships were applied to the computation of whole nerve conduction velocity distributions based on the relative number of single unit potentials of various conduction latencies required to reconstruct a recorded compound action potential. Conduction velocity distributions, which were converted to fibre diameter distributions, were in close agreement with the corresponding distributions determined histologically.

There has been considerable interest for some time, particularly among clinicians, in developing a reliable method for estimating the distribution of fibres within a whole nerve bundle. ${ }^{1-9}$ In the cou'se of studying the effects of axotomy on peripheral nerves, ${ }^{10}$ we have examined the characteristics of single unit action potentials in some detail and have applied our findings to the computation of whole nerve conduction velocity distributions. In addition, we have been able to compare these distributions to fibre diameter distributions obtained from histological examination of the same nerves.

The basic idea behind computation of conduction velocity distributions from compound action potentials originated with the work of Gasser and Grundfest. ${ }^{11}$ They attempted to reconstruct a compound action potential on the basis of the fibre diameter distribution of a nerve. Later Olson ${ }^{3}$ studied the reconstruction method in greater detail, considering a variety of factors which could influence the shape of the resultant compound action potential. Barker et $a l^{6}$ and Cummins et $a^{7}$ subsequently followed Olson's approach in formulating procedures which could be applied clinically in computing conduction velocity distributions from recorded compound action potentials. The basic assumption in their

Address for reprint requests: TE Milner, Department of Physiology, University of Alberta, Edmonton, Alberta, Canada, T6G 2 H7.

Accepted 21 January 1981 methods is that any single unit action potential can be represented by a model potential scaled according to its conduction velocity and that the compound action potential can be simply represented as a linear sum of single unit potentials. Sampling the compound action potential at $M$ discrete intervals produces a set of $\mathbf{M}$ linear simultaneous equations which can be solved to determine the conduction velocity distribution. A model single unit potential can be obtained by stimulating near the recording site on the nerve. This minimises the effects of dispersion due to differences in the conduction velocities of stimulated fibres, hence providing a template which represents the average shape of a single unit potential.

The particular formulation developed by Cummins et al $^{7}$ was chosen for the analysis of compound action potentials recorded in the experiments of our study. The mathematical theory and computer implementation are discussed in greater detail in the Appendix.

\section{Methods}

Data were obtained in a series of 26 experiments designed to determine the effects of axotomy on motor and sensory fibres in peripheral nerves. Only a brief description of the experimental procedure is given here. More detail can be found in the following paper. ${ }^{10} \mathrm{~L} 7$ and $\mathrm{S} 1$ dorsal and ventral roots and 
$\underline{A}$

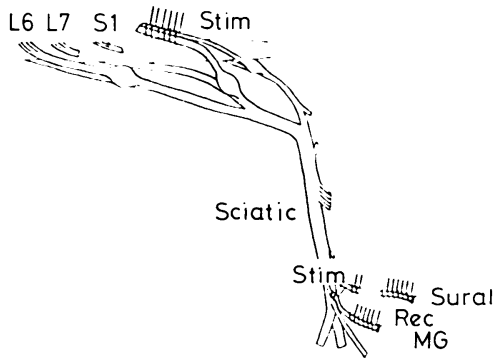

$\underline{B}$

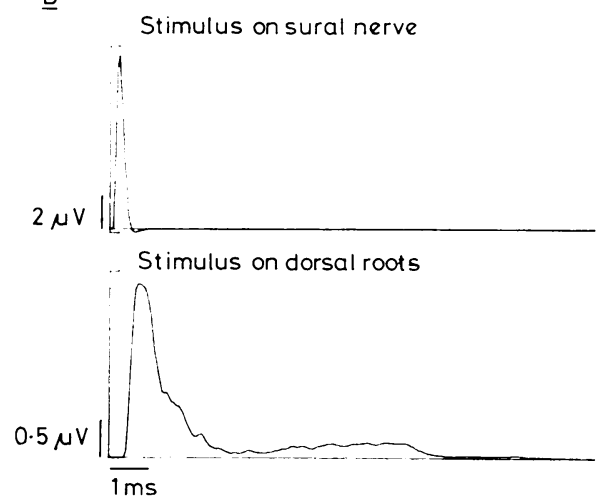

Fig 1 A Schematic representation of nerves prepared for stimulating and recording showing approximate placement of electrode arrays. B Comparison of sural nerve compound action potentials recorded while stimulating close to the recording site (upper trace) and at the dorsal roots (lower trace). The lower compound action potential has been shifted by $1.5 \mathrm{~ms}$, slightly less than the latency of the fastest conducting sural fibres. Note that it is considerably dispersed as the result of relatively large differences in the latencies of fast and slowly conducting fibres.

medial gastrocnemius (MG) and sural nerves in the cat hindlimb were prepared for stimulation and recording as illustrated in fig 1A. A six hook array $(2.5 \mathrm{~mm}$ interelectrode spacing) served both stimulating and recording purposes at the spinal roots. Nerves were ligated, cut distal to the ligature and placed on a six hook array ( $3 \mathrm{~mm}$ interelectrode spacing) in such a way that the ligature just passed over the most distal hook. A bipolar stimulating electrode consisting of two hooks was placed in contact with the nerve at a point $3-8 \mathrm{~mm}$ proximal to the first hook of the recording array. The nerve was crushed between the two most distal hooks in order that monopolar potentials could be recorded with respect to the cut end.

Before recording, the impedance was measured at each hook with respect to the most distal hook using an impedance meter with a $10 \mathrm{kHz}$ test signal. At
$10 \mathrm{kHz}$ the capacitive component of the impedance was small (phase angle less than $10^{\circ}$ ) making the impedance almost purely resistive.

Nerves and roots were stimulated with negative rectangular pulses of $0.01 \mathrm{~ms}$ duration at rates not exceeding $20 \mathrm{~Hz}$. Stimulation of the nerve at the point near the recording array produced a compound action potential with little dispersion resulting from differences in the conduction velocities of activated fibres. Stimuli delivered to the nerve were adjusted so that a near maximal response was recorded from the fastest conducting fibres, with little or no detectable contribution from slower conducting fibres. In order to minimise the stimulus artifact produced by stimulating so near the recording site, a short stretch of the MG nerve between stimulating and recording points was left attached to the main trunk of the sciatic nerve while a similar length of the sural nerve remained attached to surrounding tissue. Generally, the first one or two hooks of the recording array were grounded as well. Spinal roots were stimulated supramaximally for myelinated fibres $(10-20 \times$ threshold) in order to record the compound response of all conducting fibres. The compound action potential was dispersed considerably due to the relatively long conduction distance which accentuated the effect of conduction velocity differences. Fig 1B compares the response recorded from the sural nerve when stimulating the nerve with that obtained from dorsal root stimulation.

Recorded potentials were displayed on a storage oscilloscope and on a computer generated CRT display while being digitised and averaged. Signals were sampled and digitised using a 10 bit A/D converter. Control compound action potentials were sampled at a rate of $20 \mathrm{kHz}$. Averages normally consisted of 50 sweeps. Each potential was stored in a 256 point array.

In 11 of the 26 experiments dorsal or ventral root filaments or both were teased apart and individually stimulated in order to record single unit potentials from the nerves. The potentials were sampled at a $50 \mathrm{kHz}$ rate with averages consisting of 100-150 sweeps. At the conclusion of recording, nerve samples approximately $6-10 \mathrm{~mm}$ long were taken from the region of recording and fixed in 3\% gluteraldehyde in phosphate buffer. Experiments were normally terminated with an overdose of Nembutal. Nerves were then exposed over their entire course and conduction distances measured from lengths of thread laid along the nerve. Fixed nerve samples were rinsed three times in phosphate buffer, post-fixed in $2 \%$ osmium tetroxide in phosphate buffer for one hour, rinsed again with phosphate buffer, dehydrated in ascending alcohols and propylene oxide, then embedded in araldite. One micron thick sections were stained with $1 \%$ p-phenylenediamine and photographed with phase contrast microscopy using a green filter. The photographs were printed at $1000 \times$ magnification and fibre diameters were determined by fitting the fibre profiles to standard metric circles.

Compound action potentials and single unit 
potentials were analysed using a computer program which could determine the amplitude and half-width of a peak, as well as integrating the area under the peak. The recorded potential amplitude could be converted to dimensions of current by dividing the amplitude by the recording impedance measured at $10 \mathrm{kHz}$. Computation of the area was then equivalent to integrating current over time (giving dimensions of charge).

Another program was used to compute conduction velocity distributions. The compound action potential obtained by stimulating the nerve near the recording site served as a single unit potential template. In experiments where single unit potentials were recorded we were able to confirm that the shape of our template potential was similar to that of single unit potentials of different conduction velocities recorded from the same nerve (fig 2). We computed the relative number of single unit potentials of various conduction velocities required to reconstruct the dispersed compound action potential obtained from spinal root stimulation. The computation incorporated scaling factors which accounted for the dependence of amplitude and duration of single unit potentials on conduction velocity. These factors were determined from the accumulated single unit potential data. A description of the computer program is included in the Appendix.

\section{Results}

Approximately 200 single unit potentials were recorded from five sural nerves, 150 from five MG nerves in which dorsal roots were stimulated and another 150 from four MG nerves in which ventral roots were stimulated. Each population (single unit potentials recorded from a single nerve during one experiment) consisted of an average of 30-40 single unit potentials. Data points obtained for each nerve were plotted on a $\log$-log scale and the best-fitting straight line was computed by the least-squares method. The amplitude and integrated areas of the single unit potentials were found to correlate well as power functions of the conduction velocity (the correlation coefficient was generally greater than $\mathbf{0 . 9 5}$ in the former case and greater than 0.85 in the latter). The single unit potential half-width (a measure of the duration) did not correlate as well, although the correlation coefficient was usually greater than $0 \cdot 75$.

The relationships obtained were thus of the form $\mathrm{y}=\mathrm{kv}^{\mathrm{n}}$, where $\mathrm{k}$ is a constant, $\mathrm{v}$ the conduction velocity and $n$ the slope of the line. The combined data for sural nerves are plotted in fig 3, those for the MG dorsal root component in fig 4 and for the MG ventral root component in fig 5 . Regression parameters are listed in the table.

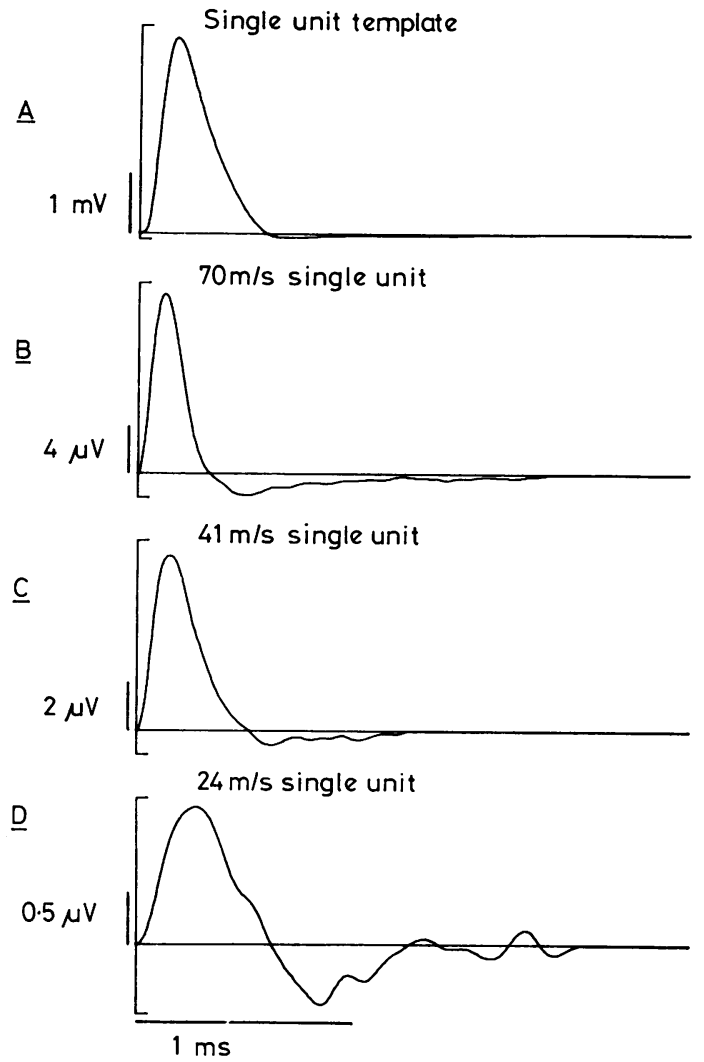

Fig $2 A$ The compound action potential obtained by stimulating an MG nerve near the site of recording to provide a template of the average shape of a single unit potential. $B, C$, and D. Single unit potentials of different conduction velocities recorded from the same nerve. Note that the positive phases of the waveforms are quite similar.

Although the bulk of the data came from single units recorded from axotomised nerves, the parameters listed in the table were used in computing the conduction velocity distributions both of control and axotomised nerves since no systematic variation with time after axotomy was apparent. The data points from various experiments are distributed more or less uniformly about the regression lines. Although the power relationships between amplitude, half-width and conduction velocity of single unit potentials are highly significant, the confidence intervals for the coefficients (k) are relatively larger than those for the exponents (n) since regression analysis estimates the logarithms of the coefficient values (table). In computing conduction velocity distributions, the amplitude coefficient simply scales the absolute 

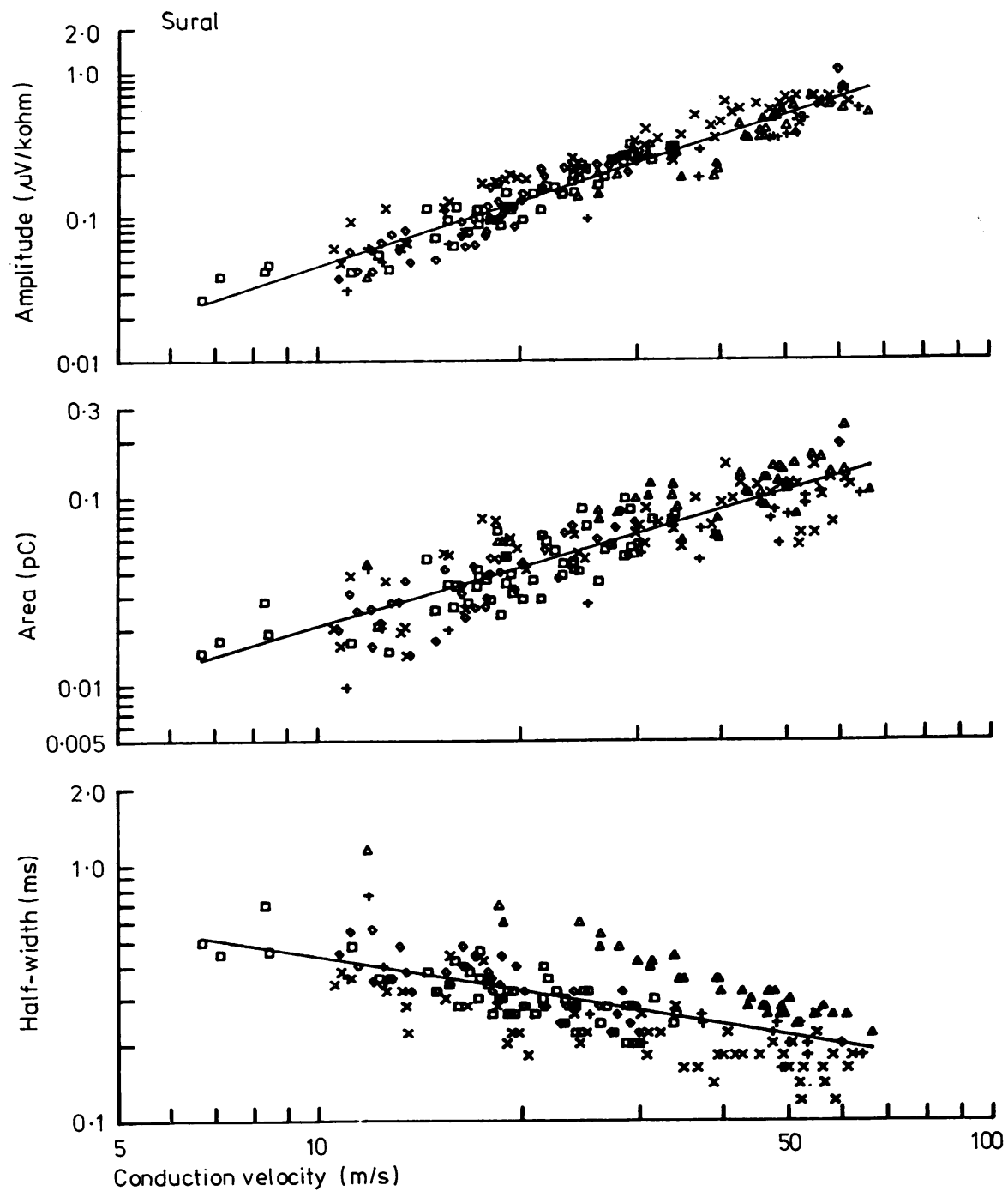

Fig 3 Single unit potential data recorded from sural nerve fibres $29(+), 35(\triangle)$, $56(\times), 198(\square)$ and $273(\diamond)$ days following axotomy plotted on logarithmic co-ardinates. In these co-ordinates the fitted straight lines represent curves of the form $y=k v^{n}$. Regression parameters are listed in the table.

magnitude of the distribution without altering its shape. The half-width coefficient has a small effect on the shape of the distribution and a relatively larger effect on its absolute magnitude. By normalising the distribution or plotting it in cumulative form much of the uncertainty can be eliminated. We also determined the total number of fibres from the computed conduction velocity distributions and although there was variability from distribution to distribution we were able to show that mean values agreed quite well with fibre counts from histological sections.

In fig $6 \mathrm{~A}$ the mean fibre diameter distribution from five control sural nerves is compared with that calculated by converting the corresponding mean conduction velocity distribution (computed as described in the Methods), to a fibre diameter distribution. Factors for converting conduction velocity to fibre diameter are based on the work of Boyd and Kalu. ${ }^{12}$ 

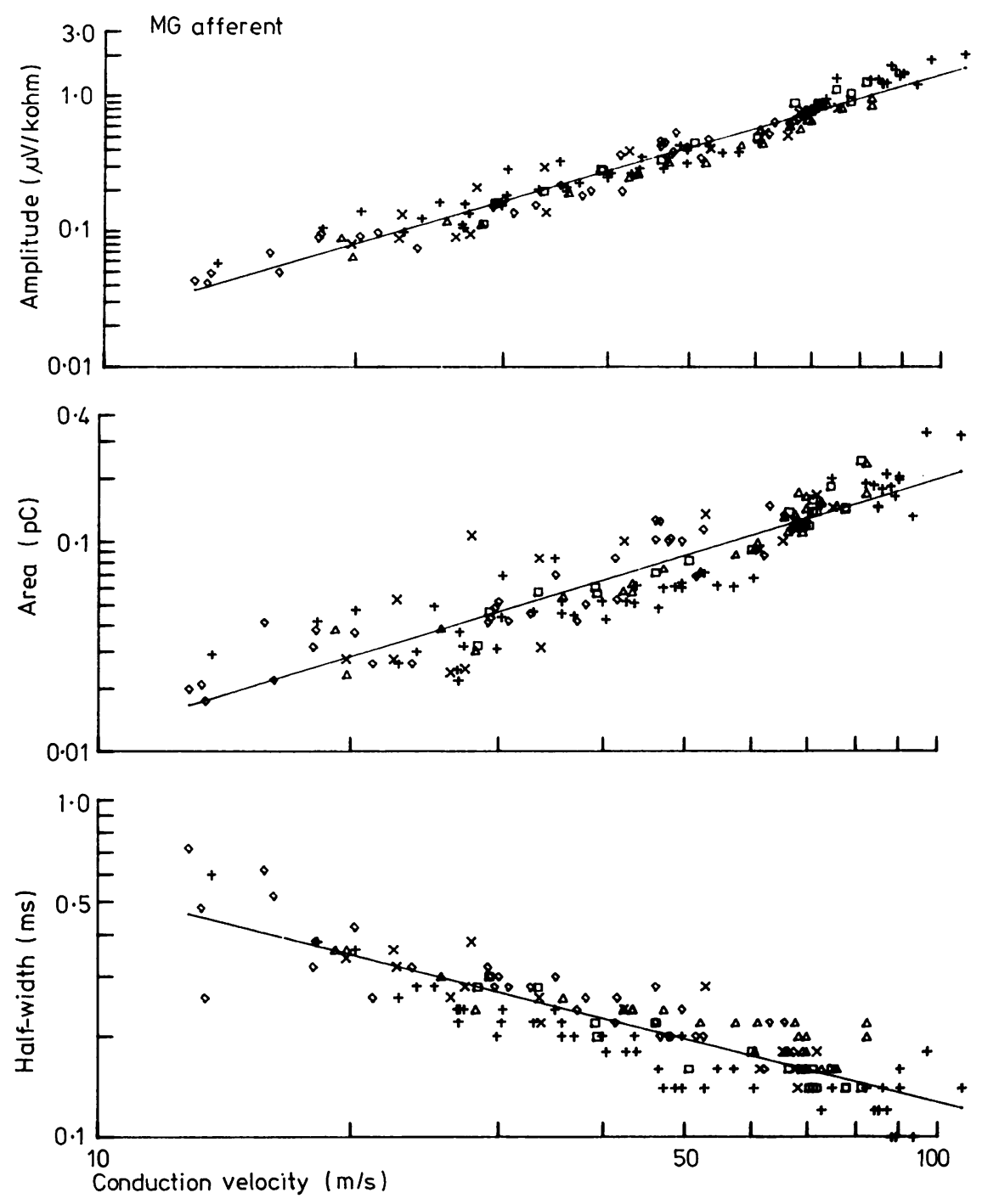

Fig 4 Single unit potential data recorded from $M G$ afferent nerve fibres $O(+), 29(\square)$, $35(\triangle), 71(\times)$ and $105(\diamond)$ days following axotomy plotted as in fig 3. Regression parameters are listed in the table.

The two distributions showed no significant differences except in the first bin. This difference was due mainly to the fact that our sampled data included only fibres conducting at velocities greater than about $10 \mathrm{~m} / \mathrm{s}$, that is fibres with diameters larger than two microns. The mean number of fibres counted in histological sections of these nerves was compared with the mean number in the computed conduction velocity distributions. There was no significant difference between these two values (two-sided $t$-test, $\mathrm{p}>0 \cdot 3$ ).
The count was $1021 \pm 94 \quad($ mean $\pm S E ; n=5)$ while the computed number was $1153 \pm 226$. A similar comparison of distributions was made for the MG nerve. Again, the two showed no significant differences except in the first bin (fig 6B). This difference was also due to the exclusion of fibres less than two microns in diameter from the sampled record. The means of the number of fibres counted $(781 \pm 52)$ and computed $(831 \pm 136)$ did not differ significantly (two-sided $t$-test, $\mathrm{p}>0.35$ ). 

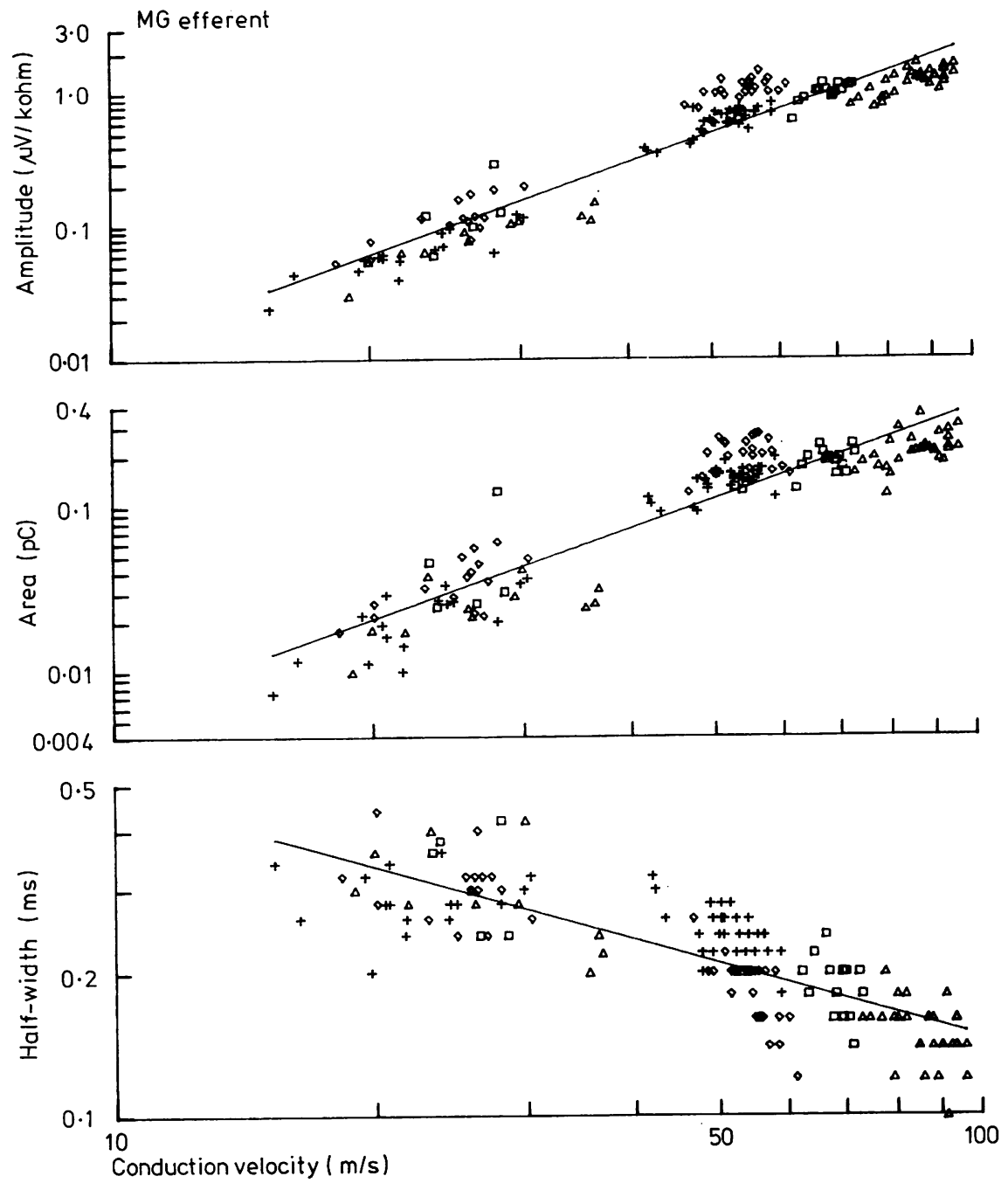

Fig 5 Single unit potential data recorded from $M G$ efferent nerve fibres $0(\Delta), 34(\diamond)$, $62(+)$ and $71(\square)$ days following axotomy plotted as in fig 3. Regression parameters are listed in the table.

\section{Discussion}

Apart from the work of Paintal, ${ }^{13}$ no systematic study of the empirical relationships between single unit potential amplitude, duration and conduction velocity of motor and sensory nerves has been undertaken to our knowledge. Paintal found an inverse relationship between action potential duration and conduction velocity, but did not attempt to fit his data with a regression line. Gasser and Grundfest, ${ }^{11}$ in studying single unit potentials of the saphenous nerve, concluded that action potential duration was approximately constant. Consequently, they assumed that the relationship between axon diameter (or equivalently conduction velocity) and amplitude had to be a linear one since such a relationship proved adequate in their reconstruction of the compound action potential from the fibre diameter distribution.

Our results for the sural nerve, a sensory nerve like the saphenous, show a different type of relationship. We found that the amplitude of single unit potentials varied approximately as conduction 
Table Regression parameters for relationships between single axon potentials and conduction velocity

\begin{tabular}{|c|c|c|c|c|c|c|}
\hline \multirow[t]{2}{*}{ Nerve } & \multirow{2}{*}{$\begin{array}{l}\text { Dependent } \\
\text { variable }\end{array}$} & \multirow[t]{2}{*}{$N$} & \multicolumn{2}{|l|}{ Coefficient } & \multirow[t]{2}{*}{ Exponent $n \pm S E$} & \multirow{2}{*}{$\begin{array}{l}\text { Correlation } \\
\text { coefficient }\end{array}$} \\
\hline & & & $\log (k) \pm S E$ & $k$ & & \\
\hline Sural & $\begin{array}{l}\text { Amplitude } \\
\text { Area } \\
\text { Half-width }\end{array}$ & $\begin{array}{l}194 \\
194 \\
194\end{array}$ & $\begin{array}{r}-2.8186 \pm 0.7007 \\
-2.7066 \pm 0.8569\end{array}$ & $\begin{array}{l}0.001518 \\
0.001966\end{array}$ & $\begin{array}{l}1.4796 \pm 0.0349 \\
1.0251 \pm 0.0426\end{array}$ & $\begin{array}{l}0.9501 \\
0.8655\end{array}$ \\
\hline \multirow[t]{2}{*}{ MG(s) } & Amplitude & 141 & $\begin{array}{r}0.0766 \pm 0.7458 \\
-3.4157 \pm 0.6780\end{array}$ & $\begin{array}{l}1 \cdot 1929 \\
0.000384\end{array}$ & $\begin{array}{r}-0.4368 \pm 0.0371 \\
1.7892 \pm 0.0344\end{array}$ & $\begin{array}{l}0.6455 \\
0.9749\end{array}$ \\
\hline & $\begin{array}{l}\text { Area } \\
\text { Half-width }\end{array}$ & 141 & $-3 \cdot 1182 \pm 0.9203$ & 0.000762 & $1 \cdot 2080 \pm 0.0467$ & 0.9090 \\
\hline \multirow[t]{3}{*}{$\mathrm{MG}(\mathrm{m})$} & Amplitude & $\begin{array}{l}141 \\
147\end{array}$ & $\begin{array}{r}0.3538 \pm 0.5830 \\
-4.1704+1.2134\end{array}$ & $2 \cdot 2589$ & $-0.6250 \pm 0.0296$ & 0.8718 \\
\hline & Area & 147 & $-4.0418 \pm 1.2803$ & $\begin{array}{l}0.000068 \\
0.000091\end{array}$ & $\begin{array}{l}2 \cdot 2707 \pm 0.0595 \\
1 \cdot 8152 \pm 0.0627\end{array}$ & $\begin{array}{l}0.9531 \\
0.9223\end{array}$ \\
\hline & Half-width & 147 & $0.1954 \pm 0.5965$ & $1 \cdot 5682$ & $-0.5155 \pm 0.0292$ & 0.8239 \\
\hline
\end{tabular}

$\mathrm{N}=$ number of cases, $\mathrm{SE}=$ standard error, $\mathrm{s}=$ sensory, $\mathrm{m}=$ motor.

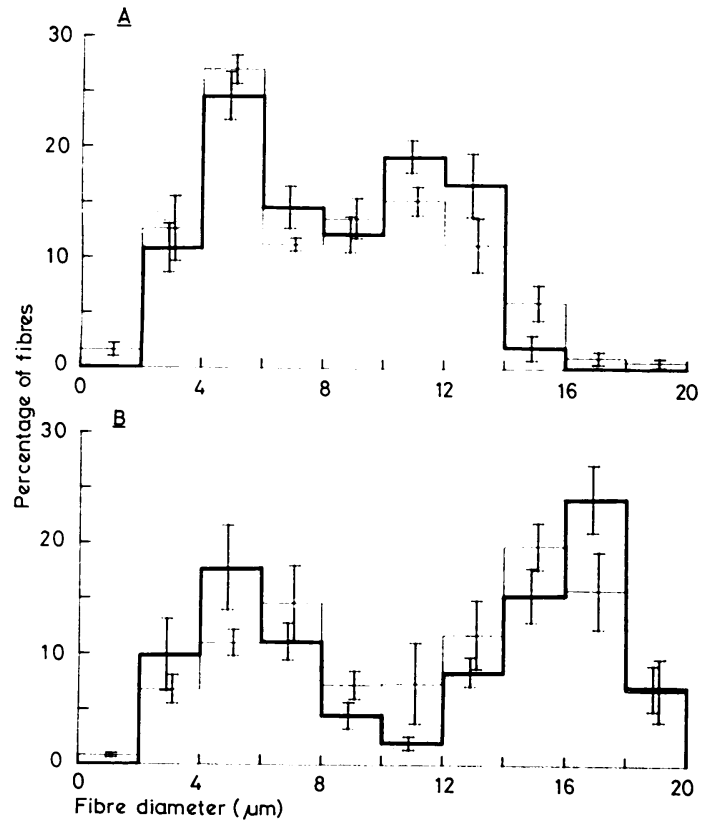

Fig 6 Comparison of the fibre diameter distributions obtained from histology (light line) with those computed from compound action potentials (heavy line). Mean distributions from 5 sural nerves $(A)$ and $5 M G$ nerves $(B)$ plotted with standard errors.

velocity raised to the power 1.5 while half-width (duration) varied approximately as the power -0.5 . If the two are combined to produce a relationship between the area of the single unit potentials and their conduction velocity (assuming the shape is roughly triangular) the result is linear. Independent measurement of the area by simple computer integration confirmed this. Gasser and Grundfest, by keeping the duration constant and varying the amplitude linearly with conduction velocity, effectively also made the integrated area of their triangular action potentials vary linearly with conduction velocity. However, it should be noted that the conduction velocity distributions computed using Gasser and Grundfest's assumption would not be identical to those which we computed since the shape of our single unit potentials was different even though the area was the same.

In examining the MG nerve we saw a relationship between half-width and conduction velocity which was similar to that observed in the sural, but the amplitude of MG single unit action potentials varied as a higher power of conduction velocity (table) which was closer to the theoretical value of two which Stein and Pearson ${ }^{14}$ predicted for unmyelinated fibres (see also Stein et $a l^{15}$ ). The values of the exponents for afferent and efferent fibres were significantly different (twosided $t$-test, $\mathrm{p}<0.0005$ ). This may have been partially due to the effects of having two widely separated populations of efferent fibres. Had alpha-motoneurones been considered alone, the amplitude exponent would have been closer to that for afferent fibres. Normally the range of alpha-motoneurone conduction velocities is quite narrow, but because of the tendency for conduction velocities to slow following axotomy we were able to extend this range. This partially filled the gap between the alpha-motoneurone and gammamotoneurone populations. Nevertheless, it is evident from fig 5 that the MG efferent single units are not normally distributed.

As was pointed out above, in comparing individual experiments no systematic change was observed in the regression parameters following axotomy. This suggests that the parameters listed in the table apply equally well to control nerves despite the fact that the bulk of the data was obtained from axotomised nerves. The reasonable agreement between distributions obtained from histology and those derived from analysis of compound action potentials recorded from the corres- 
ponding nerves bears this out and also provides a basis for using the computed conduction velocity distribution as a tool in examining the effects of axotomy on whole nerve distributions of motor and sensory fibres.

The methods described here have been applied clinically $^{6}{ }^{7}$ to a limited extent. The clinical situation is somewhat less favourable than that of the present experiments. Signals recorded from intact nerves are smaller and typically triphasic. The smaller amplitude reduces the signal to noise ratio while the triphasic nature of the recorded potentials introduces the problem of cancellation of positive and negative phases. Although cancellation may not be much of a problem in the early component of a normal compound action potential which is fairly synchronous, it will be of increasing importance when the compound action potential is more dispersed as in pathological nerves.

Experimentally, a stimulating point which is very near to the site of recording can be used to minimise dispersion in the compound potential which serves as the single unit template and another site relatively far away can be used to obtain the entire distribution of conduction velocities. Choice of stimulating and recording sites may be more limited in the clinical situation. Cummins et $a l^{8}$ have suggested an alternative estimation procedure to deal with some of these limitations. However, clinical applications so far have been restricted mainly to determination of the conduction velocity distribution of only the largest group of myelinated fibres. Whether this restriction remains will be determined as the methods are further developed.

\section{Appendix}

Computation of the conduction velocity distribution Given that the single unit potential (SUP) waveform varies with conduction velocity only and that SUPs sum linearly to produce the recorded compound action potential (CAP), the CAP may be expressed in the following form:

$$
C(t)=\sum_{i=1}^{N} w_{i} f_{i}\left(t-d_{i}\right)
$$

where $\mathrm{C}(\mathrm{t})=$ the recorded CAP as a function of time

$$
\mathrm{N}=\text { the number of fibre classes }
$$

$\mathrm{w}_{\mathrm{i}}=$ the amplitude-weighting coefficient for fibres in conduction velocity class $i$

$$
f_{i}(t)=\text { the } \begin{gathered}
\text { SUP } \\
\text { class } i
\end{gathered}
$$

$d_{i}=$ the propagation delay for fibres in class $i$

The $f_{i}(t)$ are normalised with any amplitude de- pendence on conduction velocity incorporated into the weighting coefficients $w_{1}$. The major factors determining the delay times $d_{1}$ (the time elapsed from the instant of nerve activation until the action potential arrives at the recording site) are the distance travelled along the nerve and the velocity of propagation, that is

$$
d_{1}=L / v_{1}
$$

where $\mathrm{L}=$ measured distance from the stimulating cathode to the recording site

$\mathrm{v}_{\mathbf{i}}=$ the conduction velocity represented by class $\mathrm{i}$

"Virtual" cathode effects and activation times are neglected here because the delays which they introduce are small in comparison to the conduction time which was always greater than $1 \mathrm{~ms}$.

The weighting coefficients $w_{1}$ are assumed to be of the form:

$$
\mathrm{w}_{1}=\mathrm{M}_{\mathbf{1}} \mathbf{k v}_{\mathbf{1}}{ }^{\mathrm{n}}
$$

where $M_{1}=$ the number of fibres activated in class $i$

$k=$ an empirically determined constant

$\mathrm{n}=$ an empirically determined exponent

The values of $k$ and $n$ were determined experimentally by plotting SUP amplitude against conduction velocity. The values are listed in the table. The value of $\mathrm{k}$ is not needed to determine the normalised conduction velocity distribution since it can be factored from Eqn(1) and therefore disappears upon normalisation.

The CAP model of Eqn(1) can be formulated in terms of discrete time by using equally spaced samples for the SUP and CAP functions. Eqn(1) then becomes

$$
C\left(t_{k}\right)=\sum_{i-1}^{N} w_{i} f_{i}\left(t_{k}-d_{i}\right)
$$

where $t_{k}$ is the $k$ th time point.

Assuming that there are $K$ values of the CAP, Eqn(4) may be written in matrix form.

$$
\mathrm{c}=\mathrm{Fw}
$$

where $c=a \mathrm{~K} \times 1$ column vector of $\mathrm{K}$ samples of the CAP

$F=a \quad K \times N$ matrix whose ith column is the sampled SUP function $f_{1}\left(t_{k}-d_{1}\right)$

$w=$ an $N \times 1$ column vector of the $N$ weighting coefficients

In estimating the conduction velocity distribution from a recorded CAP and known SUP properties, Eqn(5) may be viewed as a set of $K$ equations in $N$ unknowns (the $w_{1}$ ). If the matrix $F$ were square (that is $\mathrm{K}=\mathrm{N}$ ) and non-singular, the vector $\mathrm{w}$ could be determined. In general, it is not desirable to have $\mathbf{K}=\mathbf{N}$. Rather the number of time samples should exceed the number of velocity classes. ${ }^{6} 7$ The system of simultaneous linear equations is then overdetermined. A least-squares fit can be found for the 
vector $w$ by premultiplying both sides of Eqn(5) by the transpose of the Matrix F

$$
\mathbf{F}^{\mathrm{T}} \mathbf{c}=\mathrm{F}^{\mathrm{T}} \mathrm{Fw}
$$

and solving to obtain $\mathrm{w}$.

If the columns of the matrix $F$ are chosen to represent SUPs having different delay times, then they will be independent and the system will have a unique solution. Because of the symmetry of $F^{T} F$, Eqn(6) can be solved for $\mathrm{w}$ by using the square-root method. ${ }^{16}$

A FOCAL program was written to compute a conduction velocity distribution with 38 conduction velocity classes, chosen so as to have conduction latencies which differed by at least one sample period of the digitised CAP. The choice of 38 classes was somewhat arbitrary having been dictated to a large extent by the amount of computer memory available. SAPs representing individual conduction velocity classes were obtained by scaling the duration of the SUP template (see Methods) according to the experimentally determined relationship between half-width and conduction velocity. The scaling was done by linear interpolation so as to preserve the SUP waveshape. Each conduction velocity class had an associated delay time $d_{1}$ calculated from Eqn(2). In this way the sampled SUP functions $f_{1}\left(t_{k}-d_{1}\right)$ of Eqn(4) were determined and subsequently used to construct the $F$ matrix.

The program computed $F^{T} F$ and using the CAP vector $c$ found the least-squares estimate of the weighting vector $w$ by the square-root method. The weighting coefficients $w_{i}$ were then scaled by $v_{i}^{-n}$ to calculate the values of $M_{1}$ which constituted the conventional conduction velocity distribution. The $M_{1}$ were accumualted and normalised to generate the cumulative conduction velocity distribution.

This research was supported by grants from the Canadian Medical Research Council and the Muscular Dystrophy Association of Canada to RBS and by a Canadian Medical Research Council Studentship to TEM.

\section{References}

1 Buchthal F, Rosenfalck A. Evoked action potentials and conduction velocity in human sensory nerves. Brain Res 1966; 3:1-122.

2 Landau WM, Clare MH, Bishop GH. Reconstruction of myelinated nerve tract action potentials: an arithmetic method. Exp Neurol 1968; 22: 480-90.

3 Olson WH. Peripheral Nerve Compound Action Potentials and Fiber Diameter Histograms. (PhD dissertation) University of Michigan, 1973.
4 Lee RG, Ashby P, White DG, Aguayo AJ. Analysis of motor conduction velocity in human median nerve by computer simulation of compound muscle action potentials. Electroencephalogr Clin Neurophysiol 1975; 39: 225-37.

5 Leifer L, Meyer M, Morf M, Pertig B. Nerve bundle conduction velocity distribution measurement and transfer function analysis. IEEE Proceedings 1977; 65:747-55.

6 Barker AT, Brown BH, Freeston IL. Determination of the distribution of conduction velocities in human nerve trunks. IEEE Transactions on Biomedical Engineering 1979; BME-26:76-81.

7 Cummins KL, Perkel DH, Dorfman LJ. Nerve fiber conduction-velocity distributions. I. Estimation based on the single-fiber and compound action potentials. Electroencephalogr Clin Neurophysiol 1979; 46:634-46.

8 Cummins KL, Dorfman LJ, Perkel DH. Nerve fiber conduction-velocity distributions. II. Estimation based on two compound action potentials. Electroencephalogr Clin Neurophysiol 1979; 46:647-58.

9 Kovacs ZL, Johnson TL, Sax D. Estimation of the distribution of conduction velocity in peripheral nerves. Comput Biol Med 1979; 9: 281-93.

10 Milner TE, Stein RB. The effects of axotomy on the conduction of action potentials in peripheral sensory and motor nerve fibres. $J$ Neurol, Neurosurg Psychiatry 1981; 44:385-496.

11 Gasser HS, Grundfest $H$. Axon diameters in relation to the spike dimensions and the conduction velocity in mammalian A fibres. $A m J$ Physiol 1939; 127:393-414.

12 Boyd IA, Kalu KU. Scaling factor relating conduction velocity and diameter for myelinated afferent nerve fibres in the cat hind limb. $J$ Physiol (Lond) 1979; 289:277-97.

13 Paintal AS. The influence of diameter of medullated nerve fibres of cats on the rising and falling phases of the spike and its recovery. J Physiol (Lond) 1966; 184:791-811.

14 Stein RB, Pearson KG. Predicted amplitude and form of action potentials from unmyelinated nerve fibres. $J$ Theor Biol 1971; 32:539-58.

15 Stein RB, Nichols TR, Jhamandas J, Davis L, Charles D. Stable long-term recordings from cat peripheral nerves. Brain Res 1977; 128:21-38.

16 Faddeev DK, Faddeeva VN. Computational Methods of Linear Algebra. New York: WH Freeman; 1963. 